\title{
antibiotics
}

ISSN 2079-6382

www.mdpi.com/journal/antibiotics

Article

\section{Uncomplicated Urinary Tract Infections in Women in a Sao Paulo Quaternary Care Hospital: Bacterial Spectrum and Susceptibility Patterns}

\author{
Marcelo Hisano ${ }^{1}$, Homero Bruschini ${ }^{1, *}$, Antonio Carlos Nicodemo ${ }^{2}$ and Miguel Srougi ${ }^{1}$ \\ 1 Division of Urology, Hospital das Clinicas-SP, University of Sao Paulo, 255 Doutor Enéas \\ Carvalho de Aguiar Ave., 7th floor, São Paulo 05403-000, Brazil; \\ E-Mails: marcelohisano@hotmail.com (M.H.); srougi@uol.com.br (M.S.) \\ 2 Infectious Disease Department, Hospital das Clinicas-SP, University of Sao Paulo, \\ 255 Doutor Enéas Carvalho de Aguiar Ave., 4th floor, São Paulo 05403-000, Brazil; \\ E-Mail: ac_nicodemo@uol.com.br \\ * Author to whom correspondence should be addressed; E-Mail: buschini@uol.com.br; \\ Tel.: +55-11-2661-8082; Fax: +55-11-2661-8086.
}

Received: 6 January 2014; in revised form: 14 February 2014 / Accepted: 21 February 2014 / Published: 19 March 2014

\begin{abstract}
Uncomplicated urinary tract infections (UTI) in women are very common. Regular analysis of bacterial flora is important to formulate updated guidelines. The objective of this study is to determine and compare the microbiology of UTIs and their susceptibility patterns in a quaternary care hospital. In a seven-year review, the urine culture results of 480 female patients with uncomplicated UTIs were analyzed. Patients were divided into three groups according to their diagnosis and treatment characteristics: Group 1, cystitis at outpatient basis; group 2, cystitis at the Emergency Unit; and group 3, pyelonephritis. Group 1 included older patients, with a higher incidence of concomitant diabetes mellitus and recurrent UTIs. E. coli was the most common pathogen, responsible for $75.1 \%$ of cases, mainly for pyelonephritis $(87.3 \%)$. Of the oral antimicrobials tested for cystitis, amoxicillin/clavulanate and nitrofurantoin had the highest susceptibility profiles ( $84.4 \%$ and $87.3 \%$, respectively). For E. coli only, their susceptibility profiles were as high as $90.8 \%$ and $97.4 \%$, respectively. For pyelonephritis treatment, fluoroquinoles had a susceptibility profile $<90 \%$, while ceftriaxone and gentamicin had susceptibility $>90 \%$. Uncomplicated UTI treatment is becoming more challenging because the susceptibility profiles of oral antimicrobials are increasingly resistant. In our environment, cystitis can
\end{abstract}


still be managed with nitrofurantoin. Uncomplicated pyelonephritis should be managed with ceftriaxone or gentamicin.

Keywords: urinary tract infection; female; cystitis; pyelonephritis

\section{Introduction}

Urinary tract infections (UTI) in women are very common. The annual incidence was $10.8 \%$ in 2000 , and $60 \%$ of women will have at least one episode during their lifetime [1]. Additionally, after a first episode of urinary tract infection, $44 \%$ of the women will experience another episode within the following year [2].

UTI can be classified as uncomplicated or complicated. Uncomplicated UTI is defined as a UTI that occurs in a woman who has no structural or functional abnormalities, is not pregnant, and has not been instrumented [3]. Anatomically, uncomplicated UTI can be located in the lower urinary tract (cystitis) or in the upper urinary tract (pyelonephritis) [4-6].

Usually, uncomplicated cystitis can be managed on an outpatient basis in a primary care health system, while uncomplicated pyelonephritis is usually managed in a hospital setting, followed by outpatient care. At our Institution, a quaternary care hospital located in a developing country, uncomplicated cystitis can be managed either on an office outpatient basis or at the Urological Emergency Unit, according to the needs of the Public Health Care System.

As uncomplicated lower UTIs are usually treated empirically, knowledge of the contemporary flora and pattern of susceptibility is essential and mandatory. While UTI surveillance studies from Europe and the USA are available [4,7-9], equivalent studies from developing countries are sparse [10]. These data are important to formulate guidelines, particularly for such a prevalent disease, as they have the potential to change clinical practice [11]. The aim of this study was to determine the bacterial flora of uncomplicated cystitis and pyelonephritis and compare the susceptibility patterns to identify potential treatment characteristics.

\section{Experimental}

Between January, 2007, and December, 2012, we retrospectively reviewed the clinical records of 160 female patients attended at the Urological Office on an outpatient basis and 2,949 female patients treated at the Urological Emergency Unit. Of these patients, we identified and selected for analysis those with a clinical diagnosis of uncomplicated cystitis and pyelonephritis. This review had the approval of the Institutional Ethical Committee.

Uncomplicated cystitis was defined as involving clinical symptoms of dysuria, frequency, urgency, and suprapubic pain, with or without hematuria. Uncomplicated pyelonephritis was defined as clinical symptoms of flank pain, associated with nausea, vomiting, and fever $\left(>37.8^{\circ} \mathrm{C}\right)$, with or without symptoms of cystitis. All patients with these symptoms had a midstream clean catch urine culture. Urine cultures were analyzed, and a colony count of $\geq 10^{3} \mathrm{cfu} / \mathrm{mL}$ was considered positive in cases of cystitis; in the case of pyelonephritis, the threshold was $\geq 10^{4} \mathrm{cfu} / \mathrm{mL}$. Identification of isolates 
and susceptibility tests were performed on $\operatorname{VITEK}^{\circledR} 1$ and 2 automated systems (bioMérieux ${ }^{\circledR}$, Marcy L'Etoile, France). The minimal inhibitory concentrations (MICs) of antimicrobials were determined, and strains were considered susceptible, intermediately susceptible, or resistant according to the breakpoints determined by the Clinical and Laboratory Standards Institute-CLSI [12] at the time of the analysis. The following antimicrobials were tested: amikacin, amoxicillin/clavulanate $(\mathrm{A} / \mathrm{C})$, ampicillin, cefepime, cefotaxime, cefoxitin, ceftazidime, ceftriaxone, cephalothin, ciprofloxacin, gentamicin, levofloxacin, nalidixic acid, nitrofurantoin, norfloxacin, piperacillin/tazobactam (Pip/Taz), and sulfamethoxazole/trimethoprim (SMT). For some bacteria, such as Staphylococcus saprophyticus, Streptococcus agalactiae, coagulase-negative Staphylococcus, Corynebacterium spp., and yeast species, susceptibility profiles were not performed because there are no established breakpoints, according to CLSI guidelines [12].

During the review process of the record files, we were able to identify and analyze some of these three clinical characteristics: age ( $<50$ years-old and $\geq 50$ years-old), history of recurrent UTI, and presence of diabetes mellitus (DM). Recurrent UTI was defined as the presence of two or more UTI episodes during a period of six months.

We excluded patients based on the following criteria: being under 14 years-old, having kidney stones larger than $5 \mathrm{~mm}$ or any ureteral or bladder stone, having a UTI less than two months prior to the current episode, having recent urinary catheterization, being within the convalescence period of urological surgery; and having a double-J stent, urinary diversion, kidney transplantation, or any congenital urological abnormality without correction. We also excluded patients for whom urine cultures were negative or not available, despite clinical symptoms of uncomplicated UTI.

Three groups were created: group 1 -patients treated at the Urological Office as outpatients with uncomplicated cystitis; group 2-patients treated at the Urological Emergency Unit with uncomplicated cystitis; and group 3-patients treated at the Urological Emergency Unit with uncomplicated pyelonephritis. We then analyzed and compared the microbiological findings of the patients' urine samples.

Statistical analyses of the nonparametric results were performed by a chi-squared test or likelihood ratio test. When the samples were insufficient for chi-squared analysis, the Fisher exact test was performed. A $p$ value of $<0.05$ was considered statistically significant.

\section{Results}

Of the 160 patients attending the Urological Office as outpatients, 103 were included in this study with uncomplicated cystitis. Of the 2,949 patients treated at the Urological Emergency Unit, 276 were included in this study with uncomplicated cystitis and 101 with uncomplicated pyelonephritis. Seven patients in group 1, five in group 2, and one in group 3 had two episodes of UTI. The second positive samples from each of those patients were included in the analysis, giving a total of 110 urine samples in group 1, 281 in group 2, and 102 in group 3. The 480 patients included in this study provided 493 urine samples.

The mean ages (and ranges) of patients in groups 1, 2, and 3 were: 55 (18-96) years, 43.2 (15-92) years, and 36 (14-79) years, respectively. The three groups were not equivalent regarding age, history of recurrent UTI or diabetes mellitus. Group 1 had older patients, increased occurrence of recurrent UTI, and greater incidence of diabetes mellitus (Table 1). 
Table 1. Demographic data of the three groups and of the total cohort.

\begin{tabular}{|c|c|c|c|c|c|}
\hline \multirow[b]{2}{*}{ Clinical data } & \multicolumn{3}{|c|}{ Groups } & \multirow[b]{2}{*}{$\begin{array}{c}\text { Total } \\
\text { N (\%) }\end{array}$} & \multirow[b]{2}{*}{$p$} \\
\hline & $\begin{array}{c}1 \\
\text { N (\%) }\end{array}$ & $\begin{array}{c}2 \\
\text { N (\%) }\end{array}$ & $\begin{array}{c}3 \\
\mathbf{N}(\%)\end{array}$ & & \\
\hline Patients (N) & 103 & 276 & 101 & 480 & n.a. \\
\hline Age $<50$ years * & $38(36.8)$ & $172(62.3)$ & $81(81.0)$ & $291(60.6)$ & $<0.001$ \\
\hline Recurrent UTI * & $83(76.1)$ & $82(36.0)$ & $22(26.5)$ & $187(44.5)$ & $<0.001$ \\
\hline DM * & $25(22.7)$ & $18(8.5)$ & $2(2.2)$ & $45(10.9)$ & $<0.001$ \\
\hline
\end{tabular}

* Some patients with missing data; n.a.—not applicable; DM—diabetes mellitus.

The microbiology of groups 1, 2, and 3 is summarized in Table 2. In all groups, monomicrobial infection was the most common finding, varying from $92.5 \%$ to $95.1 \%$. Escherichia coli (E. coli) monoinfection was the most common microorganism in monoinfection UTI, varying from $71.2 \%$ to $87.6 \%$. Urinary infection caused by two agents (mixed infection) was identified in $7.3 \%$ of samples in group $1,7.5 \%$ in group 2 , and $4.9 \%$ in group 3 .

Table 2. Mono and mixed infections in the three groups of patients and in the total cohort.

\begin{tabular}{ccccc}
\hline Urine culture & Group 1 & Group 2 & Group 3 & Total \\
\hline Urine Samples (\%) & $110(22.3)$ & $281(57.0)$ & $102(20.7)$ & $493(100.0)$ \\
Monoinfection (\%) & $102(92.7)$ & $260(92.5)$ & $97(95.1)$ & $459(93.1)$ \\
E. coli $(\%$ monoinfection) & $78(76.5)$ & $185(71.2)$ & $85(87.6)$ & $348(75.8)$ \\
Non-E. coli $(\%$ monoinfection) & $24(23.5)$ & $75(28.8)$ & $12(12.4)$ & $111(24.2)$ \\
\hline Mixed Infection (\%) & $8(7.3)$ & $21(7.5)$ & $5(4.9)$ & $34(6.9)$ \\
E. coli $*$ (\%) & $5(62.5)$ & $13(61.9)$ & $4(80.0)$ & $22(64.7)$ \\
Non- $E$. coli $(\%$ mixed infection) & $3(37.5)$ & $8(38.1)$ & $1(20.0)$ & $12(35.3)$ \\
\hline
\end{tabular}

*E. coli as one agent.

When we analyzed all mono and mixed infection bacterial species together (Table 3), E. coli was still the most frequent pathogen, mainly for group 3 when compared to the other groups $(p=0.004)$. There was also a significantly higher frequency of Staphylococcus saprophyticus in group $2(p=0.004)$.

Table 3. Bacterial spectrum in the three groups of patients and in the entire cohort.

\begin{tabular}{|c|c|c|c|c|c|}
\hline \multirow[b]{2}{*}{ Microorganism } & \multicolumn{3}{|c|}{ Groups } & \multirow[b]{2}{*}{$\begin{array}{l}\text { Total } \\
\text { N (\%) }\end{array}$} & \multirow[b]{2}{*}{$p$} \\
\hline & $\begin{array}{c}1 \\
\text { N (\%) }\end{array}$ & $\begin{array}{c}2 \\
\text { N (\%) }\end{array}$ & $\begin{array}{c}3 \\
\text { N (\%) }\end{array}$ & & \\
\hline Escherichia coli & $83(75.5)$ & $198(70.5)$ & $89(87.3)$ & $370(75.1)$ & 0.004 \\
\hline Klebsiella pneumoniae & $7(6.4)$ & $6(2.1)$ & $6(5.9)$ & $19(3.9)$ & 0.074 \\
\hline Enterococcus faecalis & $11(10.0)$ & $18(6.4)$ & $3(2.9)$ & $32(6.5)$ & 0.113 \\
\hline Proteus mirabilis & $3(2.7)$ & $14(5.0)$ & $3(2.9)$ & $20(4.1)$ & 0.472 \\
\hline Staphylococcus saprophyticus & $3(2.7)$ & $28(10.0)$ & $2(2.0)$ & $33(6.7)$ & 0.004 \\
\hline Streptococcus agalactiae & $2(1.8)$ & $11(3.9)$ & $0(0.0)$ & $13(3.3)$ & 0.367 \\
\hline Other & $8(7.3)$ & $26(9.3)$ & $4(3.9)$ & $38(7.7)$ & 0.220 \\
\hline Total & $117(100)$ & $301(100)$ & $107(100)$ & $493(100)$ & n.a \\
\hline
\end{tabular}


We compared the susceptibility profile of E. coli to all other bacteria in groups 1, 2, and 3 (Table 4). In group 1, there was a significant difference in susceptibility for $\mathrm{A} / \mathrm{C}$, cefoxitin, nitrofurantoin and SMT. For A/C, the difference was mostly due to a decrease in the resistance profile of E. coli compared to other bacteria. Cefoxitin and nitrofurantoin had higher susceptibility for $E$. coli than for the other bacteria $(96.1 \% \times 62.5 \%$, respectively, for cefoxitin, $p=0.001 ; 97.4 \% \times 30.8 \%$, respectively, for nitrofurantoin, $p<0.001$ ). For SMT, the susceptibility of E. coli was lower than the other bacteria (56.2\% and 92.3\%, respectively; $p=0.013)$. In group 2, we found a significant difference in the susceptibility profile between $E$. coli and other bacteria for A/C $(90.8 \% \times 78.3 \%$, respectively; $p=0.031)$, cefoxitin $(96.5 \% \times 70.6 \%$, respectively; $p=0.002)$, cephalothin $(66.2 \% \times 73.1 \%$, respectively; $p=0.003)$, nitrofurantoin $(94.5 \% \times 23.8 \%$, respectively; $p<0.001)$ and SMT $(64.1 \% \times 85.2 \%$, respectively; $p=0.030$ ). In group 3, when comparing the susceptibility profile of E. coli and all other bacteria, there were significant differences in the susceptibility profiles for cefoxitin $(96.4 \% \times 66.7 \%$, respectively; $p=0.043)$ and nitrofurantoin $(95.2 \% \times 42.9 \%$, respectively; $p<0.001)$. Between groups 1,2 , and 3 , when analyzing $E$. coli, there was a susceptibility difference for amikacin $(100 \% \times 100 \% \times 92.9 \%$, respectively; $p<0.001)$; when analyzing other bacteria, there was a susceptibility difference for ciprofloxacin $(95.2 \% \times 82.5 \% \times 60 \%$, respectively; $p=0.049)$.

\section{Discussion}

E. coli is the predominant uropathogen in uncomplicated UTI, involved in $75.1 \%$ of cases. For pyelonephritis, E. coli is even more common than the other bacteria, with a frequency of $87.3 \%$; some studies have shown that E. coli is the responsible pathogen in 80 to $90 \%$ of these cases $[13,14]$. Although the genetic and behavioral risk factors for cystitis and pyelonephritis are similar, the predominance of $E$. coli causing pyelonephritis can be attributed to its intrinsic pathogenicity and virulence [15].

In general, when analyzing the susceptibility profile, there is no oral treatment option with susceptibility higher than $90 \%$, although some authors considered a threshold of $80 \%$ for inclusion in the Clinical Guidelines [4]. A/C and nitrofurantoin were closer to this level, and they are more effective, especially for E. coli. For pyelonephritis treatment, ceftriaxone had a susceptibility of at least $94 \%$; thus, it can be selected to initiate therapy in place of fluoroquinolones.

Our quaternary care hospital has its particularities. The Urological Emergency Unit treats all types of urological emergencies, from acute simple cases to complex urogenital trauma. Because it is not an exclusive referenced emergency, the UTI cases assisted there usually reflect community-acquired infections. The Urological Office takes care of more patients with comorbidities, representing more complex cases. A similar type of UTI classification has been published by Laupland et al. [16], analyzing ambulatory, hospital, and nursing home UTIs. They found E. coli frequencies of $74.2 \%$, $65.5 \%$, and $46.6 \%$ in each of these locations, respectively, as well as a difference in susceptibility profiles between them. 
Table 4. Susceptibility/resistance profiles of E. coli and other pathogens for 17 antibiotics tested in the three groups of patients and in the entire cohort.

\begin{tabular}{|c|c|c|c|c|c|c|c|c|c|c|}
\hline \multirow{3}{*}{\multicolumn{2}{|c|}{ Antibiotic }} & \multicolumn{2}{|c|}{ Group 1} & \multicolumn{2}{|c|}{ Group 2} & \multicolumn{2}{|c|}{ Group 3} & \multicolumn{2}{|c|}{ Total } & \multirow{3}{*}{$p$} \\
\hline & & \multirow{2}{*}{$\begin{array}{c}\text { Susceptible } \\
\mathbf{N}(\%)\end{array}$} & \multirow{2}{*}{$\begin{array}{c}\text { Resistant } \\
\text { N (\%) }\end{array}$} & \multirow{2}{*}{$\begin{array}{c}\text { Susceptible } \\
\text { N (\%) }\end{array}$} & \multirow{2}{*}{$\begin{array}{c}\text { Resistant } \\
\text { N (\%) } \\
\end{array}$} & \multirow{2}{*}{$\begin{array}{c}\text { Susceptible } \\
\text { N (\%) }\end{array}$} & \multirow{3}{*}{$\begin{array}{c}\text { Resistant } \\
\mathbf{N}(\%) \\
6(7.1)\end{array}$} & \multirow{2}{*}{$\begin{array}{c}\text { Susceptible } \\
\mathbf{N}(\%) \\
\end{array}$} & \multirow{2}{*}{$\begin{array}{c}\text { Resistant } \\
\mathbf{N}(\%) \\
\end{array}$} & \\
\hline & & & & & & & & & & \\
\hline AMIKACIN & E. coli & $82(100.0)$ & $0(0.0)$ & $183(100.0)$ & $0(0.0)$ & $79(92.9)$ & & $344(98.3)$ & $6(1.7)$ & $<0.001$ \\
\hline & Other & $13(100.0)$ & $0(0.0)$ & $25(96.2)$ & $1(3.8)$ & $7(100.0)$ & $0(0.0)$ & $45(97.8)$ & $1(2.2)$ & 0.56 \\
\hline & $p$ & \multicolumn{2}{|c|}{$>0.999$} & \multicolumn{2}{|c|}{0.124} & \multicolumn{2}{|c|}{$>0.999$} & \multicolumn{2}{|c|}{0.582} & \\
\hline \multirow[t]{3}{*}{$\mathbf{A} / \mathbf{C}$} & E. coli & $54(73.0)$ & $2(2.7)$ & $168(90.8)$ & $5(2.7)$ & $77(91.7)$ & $4(4.8)$ & $299(96.5)$ & $11(3.5)$ & 0.725 \\
\hline & Other & $9(69.2)$ & $3(23.1)$ & $18(78.3)$ & $4(17.4)$ & $5(71.4)$ & $2(28.6)$ & $32(78)$ & $9(22)$ & 0.81 \\
\hline & $p$ & \multicolumn{2}{|c|}{0.028} & \multicolumn{2}{|c|}{0.031} & \multicolumn{2}{|c|}{0.132} & \multicolumn{2}{|c|}{$<0.001$} & \\
\hline \multirow[t]{3}{*}{ AMPICILLIN } & E. coli & $35(42.2)$ & $48(57.8)$ & $94(48.0)$ & $100(51.0)$ & $32(42.7)$ & $41(54.7)$ & $161(46.0)$ & $189(54.0)$ & 0.577 \\
\hline & Other & $9(40.9)$ & $13(59.1)$ & $22(59.5)$ & $15(40.5)$ & $5(45.5)$ & $6(54.5)$ & $36(51.4)$ & $34(48.6)$ & 0.352 \\
\hline & $p$ & \multicolumn{2}{|c|}{0.915} & \multicolumn{2}{|c|}{0.331} & \multicolumn{2}{|c|}{0.754} & \multicolumn{2}{|c|}{0.406} & \\
\hline \multirow[t]{3}{*}{ CEFEPIME } & E. coli & $77(96.2)$ & $3(3.8)$ & $173(96.6)$ & $6(3.4)$ & $79(96.3)$ & $3(3.7)$ & $329(96.5)$ & $12(3.5)$ & 0.984 \\
\hline & Other & $13(100.0)$ & $0(0.0)$ & $25(100.0)$ & $0(0.0)$ & $6(85.7)$ & $1(14.3)$ & $44(97.8)$ & $1(2.2)$ & 0.146 \\
\hline & $p$ & \multicolumn{2}{|c|}{$>0.999$} & $>0$ & & 0.2 & & $>0$ & & \\
\hline CEFOTAXIME & E. coli & $49(94.2)$ & $3(5.8)$ & $132(95.7)$ & $6(4.3)$ & $65(97.0)$ & $2(3.0)$ & $246(95.7)$ & $11(4.3)$ & 0.755 \\
\hline & Other & $8(100.0)$ & $0(0.0)$ & $15(100.0)$ & $0(0.0)$ & $5(83.3)$ & $1(16.7)$ & $28(96.6)$ & $1(3.4)$ & 0.193 \\
\hline & $p$ & $>0$. & & $>0$ & & 0.2 & & $>0$ & & \\
\hline CEFOXITIN & E. coli & $49(96.1)$ & $0(0.0)$ & $138(96.5)$ & $2(1.4)$ & $54(96.4)$ & $2(3.6)$ & $241(98.4)$ & $4(1.6)$ & 0.268 \\
\hline & Other & $5(62.5)$ & $3(37.5)$ & $12(70.6)$ & $4(23.5)$ & $4(66.7)$ & $2(33.3)$ & $21(70.0)$ & $9(30.0)$ & 0.805 \\
\hline & $p$ & 0.0 & & 0.1 & & $\mathbf{0 . 0}$ & & $<0$. & & \\
\hline CEFTAZIDIME & E. coli & $49(94.2)$ & $3(5.8)$ & $137(95.8)$ & $6(4.2)$ & $53(96.4)$ & $3(5.4)$ & $239(95.2)$ & $12(4.8)$ & 0.880 \\
\hline & Other & $8(100.0)$ & $0(0.0)$ & $17(100.0)$ & $0(0.0)$ & $5(83.3)$ & $1(16.7)$ & $30(96.8)$ & $1(3.2)$ & 0.18 \\
\hline & $p$ & $>0$. & & $>0$ & & 0.3 & & $>0$ & & \\
\hline CEFTRIAXONE & E. coli & $30(100.0)$ & $0(0.0)$ & $58(100.0)$ & $0(0.0)$ & $31(100.0)$ & $0(0.0)$ & $119(100.0)$ & $0(0.0)$ & $>0.999$ \\
\hline & Other & $7(100.00)$ & $0(0.0)$ & $11(100.0)$ & $0(0.0)$ & $2(100.0)$ & $0(0.0)$ & $20(100.0)$ & $0(0.0)$ & $>0.999$ \\
\hline & $p$ & $>0$ & & $>0$ & & $>0$. & & $>0$. & & \\
\hline
\end{tabular}


Table 4. Cont.

\begin{tabular}{|c|c|c|c|c|c|c|c|c|c|c|}
\hline \multirow{3}{*}{\multicolumn{2}{|c|}{ Antibiotic }} & \multicolumn{2}{|c|}{ Group 1} & \multicolumn{2}{|c|}{ Group 2} & \multicolumn{2}{|c|}{ Group 3} & \multicolumn{2}{|c|}{ Total } & \multirow{3}{*}{$p$} \\
\hline & & \multirow{2}{*}{$\begin{array}{c}\text { Susceptible } \\
\mathbf{N}(\%)\end{array}$} & \multirow{2}{*}{$\frac{\text { Resistant }}{\mathbf{N}(\%)}$} & \multirow{2}{*}{$\begin{array}{c}\text { Susceptible } \\
\mathrm{N}(\%)\end{array}$} & \multirow{2}{*}{$\begin{array}{c}\text { Resistant } \\
\text { N (\%) }\end{array}$} & \multirow{2}{*}{$\begin{array}{c}\text { Susceptible } \\
\text { N (\%) }\end{array}$} & \multirow{2}{*}{$\begin{array}{c}\text { Resistant } \\
\mathbf{N}(\%) \\
\end{array}$} & \multirow{2}{*}{$\begin{array}{c}\text { Susceptible } \\
\mathbf{N}(\%) \\
\end{array}$} & \multirow{2}{*}{$\begin{array}{c}\text { Resistant } \\
\text { N (\%) } \\
\end{array}$} & \\
\hline & & & & & & & & & & \\
\hline CEPHALOTIN & E. coli & $56(67.5)$ & $18(21.7)$ & $129(66.2)$ & $28(14.4)$ & $59(66.3)$ & $13(14.6)$ & $244(80.5)$ & $59(19.5)$ & 0.479 \\
\hline & Other & $9(60.0)$ & $5(33.3)$ & $19(73.1)$ & $7(26.9)$ & $6(75.0)$ & $2(25.0)$ & $34(70.8)$ & $14(29.2)$ & 0.813 \\
\hline & $p$ & \multicolumn{2}{|c|}{0.601} & \multicolumn{2}{|c|}{0.003} & \multicolumn{2}{|c|}{0.179} & \multicolumn{2}{|c|}{0.124} & \\
\hline \multirow[t]{3}{*}{ CIPROFLOXACIN } & E. coli & $63(75.9)$ & $19(22.9)$ & $164(83.2)$ & $32(16.2)$ & $75(84.3)$ & $14(15.7)$ & $302(82.3)$ & $65(17.7)$ & 0.337 \\
\hline & Other & $20(95.2)$ & $1(4.8)$ & $33(82.5)$ & $5(12.5)$ & $6(60.0)$ & $4(40.0)$ & $59(85.5)$ & $10(14.5)$ & 0.049 \\
\hline & $p$ & \multicolumn{2}{|c|}{0.081} & \multicolumn{2}{|c|}{0.136} & \multicolumn{2}{|c|}{0.080} & \multicolumn{2}{|c|}{0.516} & \\
\hline \multirow[t]{3}{*}{ GENTAMICIN } & E. coli & $77(92.8)$ & $5(6.0)$ & $184(94.4)$ & $10(5.1)$ & $87(97.8)$ & $1(1.1)$ & $348(95.6)$ & $16(4.4)$ & 0.143 \\
\hline & Other & $13(86.7)$ & $2(13.3)$ & $31(96.9)$ & $0(0.0)$ & $10(100.0)$ & $0(0.0)$ & $54(96.4)$ & $2(3.6)$ & 0.065 \\
\hline & $p$ & \multicolumn{2}{|c|}{0.555} & \multicolumn{2}{|c|}{0.105} & \multicolumn{2}{|c|}{0.806} & \multicolumn{2}{|c|}{$>0.999$} & \\
\hline \multirow[t]{3}{*}{ LEVOFLOXACIN } & E. coli & $58(78.4)$ & $15(20.3)$ & $156(84.3)$ & $27(14.6)$ & $72(85.7)$ & $11(13.1)$ & $286(84.4)$ & $53(15.6)$ & 0.406 \\
\hline & Other & $13(92.9)$ & $1(7.1)$ & $21(84.0)$ & $3(12.0)$ & $5(62.5)$ & $3(37.5)$ & $39(84.8)$ & $7(15.2)$ & 0.186 \\
\hline & $p$ & 0.3 & & 0.5 & & 0.2 & & & & \\
\hline NALIDIXIC ACID & E. coli & $52(70.3)$ & $22(29.7)$ & $149(81.0)$ & $35(19.0)$ & $66(80.5)$ & $16(19.5)$ & $267(78.5)$ & $73(21.5)$ & 0.147 \\
\hline & Other & $12(92.3)$ & $1(7.7)$ & $19(82.6)$ & $4(17.4)$ & $5(71.4)$ & $2(28.6)$ & $36(83.7)$ & $7(16.3)$ & 0.466 \\
\hline & $p$ & 0.1 & & $>0$. & & 0.6 & & & & \\
\hline NITROFURANTOIN & E. coli & $74(97.4)$ & $0(0.0)$ & $172(94.5)$ & $3(1.6)$ & $80(95.2)$ & $1(1.2)$ & $326(98.8)$ & $4(1.2)$ & 0.344 \\
\hline & Other & $4(30.8)$ & $5(38.5)$ & $5(23.8)$ & $13(61.9)$ & $3(42.9)$ & $4(57.1)$ & $12(35.3)$ & $22(64.7)$ & 0.621 \\
\hline & $p$ & $<0.0$ & & $<0$. & & $<\mathbf{0 .}$ & & & & \\
\hline NORFLOXACIN & E. coli & $26(83.9)$ & $5(16.1)$ & $54(85.7)$ & $8(12.7)$ & $25(83.3)$ & $5(16.7)$ & $105(85.4)$ & $18(14.6)$ & 0.859 \\
\hline & Other & $10(83.3)$ & $2(16.7)$ & $17(77.3)$ & $3(13.6)$ & $3(75.0)$ & $1(25.0)$ & $30(83.3)$ & $6(16.7)$ & 0.896 \\
\hline & $p$ & $>0.9$ & & 0.3 & & 0.5 & & & & \\
\hline PIP/TAZ & E. coli & $47(94.0)$ & $8(100.0)$ & $133(98.5)$ & $0(0.0)$ & $52(100.0)$ & $0(0.0)$ & $232(98.7)$ & $3(1.3)$ & 0.009 \\
\hline & Other & $3(6.0)$ & $0(0.0)$ & $16(94.1)$ & $1(5.9)$ & $6(100.0)$ & $0(0.0)$ & $30(96.8)$ & $1(3.2)$ & 0.541 \\
\hline & $p$ & $>0.9$ & & 0.0 & & $>0$. & & & & \\
\hline SMT & E. coli & $45(56.2)$ & $35(43.8)$ & $118(64.1)$ & $66(35.9)$ & $55(64.7)$ & $30(35.3)$ & $218(62.5)$ & $131(37.5)$ & 0.424 \\
\hline & Other & $12(92.3)$ & $1(7.7)$ & $23(85.2)$ & $4(14.8)$ & $7(100.0)$ & $0(0.0)$ & $42(89.4)$ & $5(10.6)$ & 0.341 \\
\hline & $p$ & 0.0 & & 0.0 & & 0.0 & & & & \\
\hline
\end{tabular}

Note: Intermediate susceptible corresponds to $100 \%$ (susceptible + resistant). A/C-amoxicillin/clavulanic acid; Pip/Taz-piperacillin/tazobactam; SMT-sulfamethoxazole/trimethoprim. 
In 2008, a multi-center analysis of the microbiology of uncomplicated cystitis (ARESC) [8] showed an E. coli frequency of $76.7 \%$, close to that found in the present study. The overall susceptibilities to ampicillin, A/C, ciprofloxacin, nalidixic acid, and nitrofurantoin were $45.1 \%, 82.1 \%, 91.8 \%, 81.4 \%$, and $95.2 \%$, respectively. In the same study, the susceptibility profiles of Brazilian samples for ampicillin, A/C, ciprofloxacin, nalidixic acid, and nitrofurantoin were 33.8\%, 78.7\%, 89.0\%, 74.7\%, and $84.1 \%$, respectively. In our study considering both groups 1 and 2 together, we found susceptibilities of $47.3 \%, 84.4 \%, 82.1 \%, 78.9 \%$, and $87.3 \%$, respectively, for the same antimicrobials. Our results showed susceptibility profiles for these antimicrobials similar to those found for Brazilian samples, although the period and place of analysis were different (the ARESC study was conducted between September, 2003 and June, 2006 and involved four Brazilians centres).

When we compared E. coli to the other bacteria (mainly Klebsiella pneumonia, Enterococcus faecalis and Proteus mirabilis) in all groups, the susceptibility profiles differed, mainly for A/C, cefoxitin, and nitrofurantoin. These antimicrobials had higher susceptibility results when the UTI agent was E. coli. In another study of the ARESC group [17], the susceptibility profiles of Brazilian E. coli samples for ampicillin, A/C, ciprofloxacin, nalidixic acid, nitrofurantoin, and SMT were 37.7\%, $79.8 \%, 89.2 \%, 75.4 \%, 94.3 \%$, and $54.5 \%$, respectively. For the same antimicrobials, our study found susceptibilities of $46.0 \%, 96.5 \%, 82.3 \%, 78.5 \%, 98.8 \%$, and $62.5 \%$, respectively. A similar result was found by Linhares et al. [18] for nitrofurantoin and pivmecillinam, with the same pattern of high susceptibility for $E$. coli but low for non-E. coli, although they analyzed male and female patients. Nitrofurantoin is known to have no activity against Proteus spp. and Pseudomonas aeruginosa [19]; this information is in line with our results because $P$. mirabilis was analyzed as a non- $E$. coli agent. Interestingly, although SMT had a low susceptibility profile for E. coli $(56.2 \%, 64.1 \%$, and $64.7 \%$ for groups 1,2 , and 3 , respectively), it had a higher susceptibility profile to non- $E$. coli agents, reaching $92.3 \%, 85.2 \%$, and $100 \%$ susceptibility in groups 1,2 , and 3 , respectively ( $p<0.05$ for groups 1 and 2 only). These findings may indicate that SMT cannot be considered for E. coli treatment but can be used for non-E. coli bacteria, although others studies did not find similar results $[17,18]$.

Our study has some limitations. The retrospective nature of this analysis has an intrinsic bias. As we retrospectively analyzed six years of uncomplicated UTI, some changes in MIC occurred during these years according to CLSI. We are aware of this fact, but as we considered final outcomes of the test (Susceptible, Intermediately Susceptible, and Resistant) to treat the patients at that moment successfully, we considered it clinically relevant; other groups had already utilized this classification for the results of susceptibility tests $[10,16,18]$. Another limitation is the fact that susceptibility profiles were not performed for some bacteria.

The three groups were not statistically similar; group 1 had older patients with higher incidences of diabetes mellitus and recurrent UTI than groups 2 and 3. This difference adds another source of bias to this study, however, as already mentioned, this difference was due to our local health care organization. Additionally, confirming our perception, this group division resembled a comparison between uncomplicated UTIs from a quaternary care hospital (group 1) and community-acquired uncomplicated UTIs (groups 2 and 3).

The 2013 European Guideline on urological infections [5] outlines the antimicrobials of choice for the treatment of uncomplicated cystitis: fosfomycin trometamol, pivmecillinam and nitrofurantoin. Our study did not test fosfomycin trometamol or pivmecillinam; the latter is not available in our country. 
Fosfomycin trometamol is not routinely tested at our hospital because, at the time of urine culture analysis, there were no established MIC breakpoints for some bacteria and antimicrobials according to CLSI guidelines [12]. Additionally, due to the retrospective nature of this study, we were unable to perform retests. Nitrofurantoin had a general susceptibility profile close to $90 \%$, but for $E$. coli, it was at least $94.5 \%$. In our environment, $\mathrm{A} / \mathrm{C}$ and ciprofloxacin had susceptibilities of $84.4 \%$ and $82.1 \%$, respectively; both were at the lower limit for inclusion as first options for cystitis treatment. Although some European countries reported similar susceptibility profiles, these antibiotics are recommended by their national guidelines [20].

For uncomplicated pyelonephritis, the Infectious Disease Society of America Guidelines [4] and the 2013 European Guideline [5] recommend fluoroquinolones as alternative therapeutic agents. Our study showed a susceptibility profile lower than $90 \%$ for these antimicrobials, making them unsuitable for our environment because the susceptibility threshold to be considered as a first line therapy should be higher than $90 \%[4,6]$. The alternatives for empirical antimicrobial therapy are ceftriaxone, with a susceptibility of $100 \%$, or gentamicin, with a susceptibility of $98.0 \%$. Although nitrofurantoin had an acceptable susceptibility profile for pyelonephritis treatment, mainly for E. coli, due to its rapid renal excretion and insufficient therapeutic blood level, it is not indicated to treat pyelonephritis [21].

\section{Conclusions}

Uncomplicated UTI treatment is becoming more difficult to manage because the susceptibility profiles of current oral antimicrobials are becoming more resistant. For simple cystitis, nitrofurantoin is at the lower limit of acceptable susceptibility to continue to be an antimicrobial of choice, but it is still very active for E. coli, as well as A/C. For pyelonephritis, fluoroquinolones are not suitable as a first line therapy and should be replaced by ceftriaxone or gentamicin. Judicious use of antimicrobials, adhesion to guidelines, and new drug alternatives should be considered as strategies to avoid increasing resistance.

\section{Acknowledgments}

The authors thank Elisa Cruz for her helpful assistance and Rogério Ruscitto for his statistical analysis assistance.

\section{Author Contributions}

Marcelo Hisano worked on collecting data and preparing the manuscript. Homero Bruschini worked on collecting data, preparing and reviewing the manuscript. Antonio Carlos Nicodemo worked on preparing and reviewing the manuscript. Miguel Srougi worked on preparing and reviewing the manuscript.

\section{Conflicts of Interest}

The authors declare no conflicts of interest. 


\section{References}

1. Foxman, B.; Barlow, R.; D’Arcy, H.; Gillespie, B.; Sobel, J.D. Urinary tract infection: Self-reported incidence and associated costs. Ann. Epidemiol. 2000, 10, 509-515.

2. Ikäheimo, R.; Siitonen, A.; Heiskanen, T.; Kärkkäinen, U.; Kuosmanen, P.; Lipponen, P.; Mäkelä, P.H. Recurrence of urinary tract infection in a primary care setting: Analysis of a 1-year follow-up of 179 women. Clin. Infect. Dis. 1996, 22, 91-99.

3. Foxman, B. The epidemiology of urinary tract infection. Nat. Rev. Urol. 2010, 7, 653-660.

4. Gupta, K.; Hooton, T.M.; Naber, K.G.; Wullt, B.; Colgan, R.; Miller, L.G.; Moran, G.J.; Nicolle, L.E.; Raz, R.; Schaeffer, A.J.; et al. International clinical practice guidelines for the treatment of acute uncomplicated cystitis and pyelonephritis in women: A 2010 update by the Infectious Diseases Society of America and the European Society for Microbiology and Infectious Diseases. Clin. Infect. Dis. 2011, 52, e103-e120.

5. Grabe, M.; Bjerklund-Johansen, T.E.; Botto, H.; Çek, M.; Naber, K.G.; Pickard, R.S.; Tenke, P.; Wagenlehner, F.; Wullt, B. EAU Guidelines on Urological Infection. 2013. Available online: http://www.uroweb.org/gls/pdf/18_Urological\%20infections_LR.pdf (accessed on 30 December 2013).

6. Colgan, R.; Williams, M.; Johnson, J.R. Diagnosis and treatment of acute pyelonephritis in women. Am. Fam. Physician 2011, 84, 519-526.

7. Kahlmeter, G. An international survey of the antimicrobial susceptibility of pathogens from uncomplicated urinary tract infections: the ECO-SENS Project. J. Antimicrob. Chemother. 2003, $51,69-76$.

8. Naber, K.G.; Schito, G.; Botto, H.; Palou, J.; Mazzei, T. Surveillance study in Europe and Brazil on clinical aspects and Antimicrobial Resistance Epidemiology in Females with Cystitis (ARESC): Implications for empiric therapy. Eur. Urol. 2008, 54, 1164-1175.

9. Kahlmeter, G.; Poulsen, H.O. Antimicrobial susceptibility of Escherichia coli from community-acquired urinary tract infections in Europe: The ECO·SENS study revisited. Int. J. Antimicrob. Agents 2012, 39, 45-51.

10. Kiffer, C.R.; Mendes, C.; Oplustil, C.P.; Sampaio, J.L. Antibiotic resistance and trend of urinary pathogens in general outpatients from a major urban city. Int. Braz. J. Urol. 2007, 33, 42-48.

11. Masterton, R. The importance and future of antimicrobial surveillance studies. Clin. Infect. Dis. 2008, 47, S21-S31.

12. Clinical and Laboratory Standards Institute. Performance standards for antimicrobial susceptibility testing; seventeenth to twenty-second informational supplement. CLSI document M100-S17-22. Clinical and Laboratory Standards Institute: Wayne, PA, USA, 2007-2012.

13. Czaja, C.A.; Scholes, D.; Hooton, T.M.; Stamm, W.E. Population-based epidemiologic analysis of acute pyelonephritis. Clin. Infect. Dis. 2007, 45, 273-280.

14. Nicolle, L.E. Uncomplicated urinary tract infection in adults including uncomplicated pyelonephritis. Urol. Clin. North Am. 2008, 35, 1-12.

15. Wiles, T.J.; Kulesus, R.R.; Mulvey, M.A. Origins and virulence mechanisms of uropathogenic Escherichia coli. Exp. Mol. Pathol. 2008, 85, 11-19.

16. Laupland, K.B.; Ross, T.; Pitout, J.D.; Church, D.L.; Gregson, D.B. Community-onset urinary tract infections: A population-based assessment. Infection 2007, 35, 150-153. 
17. Schito, G.C.; Naber, K.G.; Botto, H.; Palou, J.; Mazzei, T.; Gualco, L.; Marchese, A. The ARESC study: An international survey on the antimicrobial resistance of pathogens involved in uncomplicated urinary tract infections. Int. J. Antimicrob. Agents 2009, 34, 407-413.

18. Linhares, I.; Raposo, T.; Rodrigues, A.; Almeida, A. Frequency and antimicrobial resistance patterns of bacteria implicated in community urinary tract infections: A ten-year surveillance study (2000-2009). BMC Infect. Dis. 2013, 13, e19.

19. Wagenlehner, F.M.; Weidner, W.; Naber, K.G. Antibiotics in urology: New essentials. Urol. Clin. North Am. 2008, 35, 69-79.

20. Haslund, J.M.; Dinesen, M.R.; Nielsen, A.B.S.; Llor, C.; Bjerrum, L. Different recommendations for empiric first-choice antibiotic treatment of uncomplicated urinary tract infections in Europe. Scand. J. Prim. Health Care 2013, 31, 235-240.

21. Shepherd, A.K.; Pottinger, P.S. Management of urinary tract infections in the era of increasing antimicrobial resistance. Med. Clin. North Am. 2013, 97, 737-757.

(C) 2014 by the authors; licensee MDPI, Basel, Switzerland. This article is an open access article distributed under the terms and conditions of the Creative Commons Attribution license (http://creativecommons.org/licenses/by/3.0/). 